\title{
Development cutting plane method to find optimal integer solution for Fuzzy linear programming problems
}

\author{
Isra'a H. Hassan ${ }^{1}$, Rasha J. Mitilf ${ }^{2}$ and Waleed Khalid Jaber ${ }^{3}$ \\ ${ }^{1}$ University of Technology-Iraq Engineering Communications, Iraq \\ ${ }^{2}$ University of Technology-Iraq Applied Sciences, Iraq \\ ${ }^{3}$ University of Thi-Qar, Iraq Computer Science \& Mathematics, Iraq
}

Received: 28 April 2019, Accepted: 8 July 2019

Published online: 29 September 2019.

\begin{abstract}
An integer linear programming problems (ILPP) in which coefficient of objective function are Fuzzy Number and Completely Fuzzy Integer Linear Programming Problems (CFILPP) may be treated. This work presents a new technique to get the optimal integer solution for linear programming problems with fuzzy variables by using Ranking function. Examples were presented they clarifying the accuracy of the reviewed technique. This technique is the best to find the optimal integer solution of (FILPP).
\end{abstract}

Keywords: Operation research, Plane Method, Fuzzy Linear Programming Problems.

\section{Introduction}

(LP) is an almost important mechanism for operation research. It is involved with the linear function while satisfying a set of linear equality and inequality constraints. Fuzzy set theory has been used many people discipline like control theory and administration science, arithmetic modeling and manufacturing application [1] such as analysis of water, air pollution regulation problem the idea fuzzy linear programming (LP) was the first time by zimmermann [6]. (FLPP) have a fundamental role in fuzzy modeling which can subedit uncertainly in realistic environment [6]. Afterward, many authors have treated differences kinds of the (FLPP) and submit several approaches several these problems.

Specially, the appropriate methods are established on the idea of comparison of fuzzy numbers with help of ranking functions [3],[7],[8]. (LP) problems are related to many fields, such as artificial intelligence and operations research. It is important to representing the display of problems [1].Finally, Herrera and Verdegay [5] have approach three methods for solving three designs of (FILP) established on the representation theorem and on fuzzy number ranking method for a (FLIP) problem by crisp linear integer programming problems. Pandian and Jayalakshmi [1] have suggested a model method for solution (LIP) problem with fuzzy variables by using the traditional (LIP). A new technique was introduced in this work for finding an optimal fuzzy integer solution for (FILP) problems, when the coefficients of objective function are fuzzy numbers, and (CFILPP) by development cutting plane method and integer simples tableau with help of ranking function, the obtained results exactly satisfy all the constraints and the computation in the proposed method is more easy and simple. 


\section{Preliminaries}

Definition 1. [4] If $X$ is a collection of objects denoted generically by $X$, then a fuzzy set $\tilde{a}$ in $X$ is defined to be a set of ordered pairs $\tilde{a}=\left\{\left(x, \mu_{\tilde{a})}: x \in X\right\}\right.$, where $\mu_{\tilde{a}}$ is called the membership function for the fuzzy set. The membership function maps each element of $X$ value between $[0,1]$.

Definition 2. [2] A fuzzy set ã, defined on the universal set of real numbers $R$, is said to be a fuzzy number if its membership function has the following characteristics.

(i) $M_{\tilde{a}}: R \rightarrow[0,1]$ is continuous.

(ii) $M_{\tilde{a}}(x)=0, \forall x \in(-\infty, a] \bigcup[d, \infty)$

(iii) $M_{\tilde{a}}(x)$ is strictly increasing on $[a, b]$ and strictly decreasing on $[c, d]$

(iv) $M_{\tilde{a}}(x)=1 \forall x \in[a, b]$ where $a \leq b \leq c \leq d$.

Definition 3. [1],[4],[9] For any fuzzy number $\tilde{a}=\left(a^{l}, a^{u}, \alpha, \beta\right)$ the trapezoidal membership function is as follows

$$
M_{\tilde{a}}(x) \begin{cases}1-\frac{a^{l}-x}{\alpha}, & a^{l}-\alpha \leq x<a^{l} \\ 1, & a^{l} \leq x \leq a^{u} \\ 1-\frac{x-a^{u}}{\beta}, & a^{u} \leq x \leq a^{u}+\beta \\ 0, & \text { otherwise. }\end{cases}
$$

\section{Ranking function [4]}

The Ranking function is applicable approach for ordering fuzzy number. The Ranking function is denoted by $F(\Re)$ where $\mathfrak{R}: F(\mathfrak{R}) \rightarrow \mathfrak{R}$, is the set of fuzzy numbers. Let $\tilde{a}=\left(a^{l}, a^{u}, \alpha, \beta\right) \in F(\mathfrak{R})$ then $\mathfrak{R}(\tilde{a})=\frac{1}{2}\left(a^{l}+a^{u}+\frac{1}{2}(\beta-\alpha)\right)$.

\section{Fuzzy integer linear programming problems (FILPP) [1]}

A fuzzy linear integer programming (FILP) is defined as

$$
\begin{aligned}
& \left(P_{1}\right)\left(\begin{array}{l}
\text { Max } \\
\text { or } \\
\operatorname{Min},
\end{array}\right) \tilde{Z}=c \tilde{x} \\
& \text { subject to } A \tilde{x} R^{(\leq, \approx, \geq)} \tilde{b} \\
& \tilde{x} \geq 0 \text { are integer }
\end{aligned}
$$

where $A=\left(a_{i j}\right)_{m * n}$ is nonnegative matrix, the cost vector $c \in\left(F(R)^{n}\right)$ and $\tilde{x}=\left(\tilde{x}_{j}\right)_{n * 1}$ and $\tilde{b}=\left(\tilde{b}_{i}\right)_{m * 1}$ are non-negative real fuzzy vectors. $\forall 1 \leq j \leq n, 1 \leq i \leq m$

Definition 4. [2] (Feasible and Optimal Solution) A fuzzy vector $\tilde{x}$ is said to be a feasible solution of the problem $\left(P_{1}\right)$ if $\tilde{x}$ satisfies $A \tilde{x} R^{(\leq, \approx, \geq)} \tilde{b}$ and $\tilde{x} \geq 0$

$x_{0}$ is optimal feasible solutions to the problem $\left(P_{1}\right)$ if $z-\tilde{c} x_{0} \geq 0$. 


\section{Completely fuzzy integer linear programming problems (CFILPP) [7]}

Consider the following (CFILPP) with (m) fuzzy constraints and (n) fuzzy variables as follows:

$$
\begin{gathered}
\left(P_{2}\right)\left(\begin{array}{l}
\text { Max } \\
\text { or } \\
\text { Min, }
\end{array}\right) \tilde{Z}=\tilde{c}^{T} \tilde{x} \\
\text { subject to } A \tilde{x} \leq \tilde{b} \\
\tilde{x} \geq 0 \text { are integer }
\end{gathered}
$$

where $\tilde{c}=\left(\tilde{c}_{j}\right)_{1 * n} \tilde{A}=\left(\tilde{a}_{i j}\right)_{m * n}, \tilde{X}=\left(\tilde{x}_{j}\right)_{n * 1}$ and $\tilde{b}=\left(\tilde{b}_{i}\right)_{m * 1} \in F(R) \quad \forall 1 \leq j \leq n, 1 \leq i \leq m$ In this work we solve two cases of FILPP. The first case are fuzzy numbers in the objective function coefficients, the second case are completely fuzzy integer linear programming problems.

\section{Development cutting plane method for (FILPP)}

Now we suggest a new technique to find an optimal fuzzy integer linear solution for (FILPP) with help of the development of simplex tableau as shown in the following examples

$$
\operatorname{Max} \tilde{z} R^{=} \tilde{C}_{B} X_{B}+\tilde{C}_{N} S_{N}
$$

Subject to

$$
\begin{gathered}
B X_{B}+N S_{N} R^{=} b \\
X_{B} \geq 0, S_{B} \geq 0 \text { are all integers }
\end{gathered}
$$

Hence, $X_{B}+B^{-1} N S_{N}=B^{-1} b$. Therefore

$$
\begin{gathered}
\tilde{Z}=\tilde{C}_{B}\left(B^{-1} b-B^{-1} N S_{N}\right)+\tilde{C}_{N} S_{N} \\
\tilde{C}_{B} B^{-1} b-\tilde{C}_{B} B^{-1} N S_{N}+\tilde{C}_{N} S_{N} \\
\tilde{Z}+\left(\tilde{C}_{B} B^{-1} N-\tilde{C}_{N}\right) X_{N}=\tilde{C}_{B} B^{-1} b
\end{gathered}
$$

at $S_{N}=0$, we have $X_{B}=B^{-1} b$, and $\tilde{Z}=\tilde{C}_{B} B^{-1} b$

Then the above problem can be solved by using development integer simplex tableau as below: Then Apply simplex

\begin{tabular}{|c|c|c|}
\hline Basic & $-t_{n}$ & R.H.S \\
\hline$\tilde{Z}$ & $\tilde{C}_{B} B^{-1} N-\tilde{C}_{N}$ & $\tilde{C}_{B} B^{-1} b$ \\
\hline$X_{B}$ & $-\mathrm{I}$ & 0 \\
$S_{N}$ & $B^{-1} N$ & $B^{-1} b$ \\
\hline$S c_{i}$ & {$\left[\frac{\text { pivotrow }}{\text { pivotelement }}\right]$} & \\
\hline
\end{tabular}

algorithm, the entering variable can be found by use ranking function. [.] denoted the integer function. 


\section{Numerical example}

To demonstrate the solution process for (FNILPP) by using development cutting plane method and integer simplex tableau. let us treat the following problem

$$
\begin{gathered}
\operatorname{Max} Z=(5,8,2,5) x_{1}+(6,10,2,6) x_{2} \\
2 x_{1}+3 x_{2} \leq 6 \\
5 x_{1}+4 x_{2} \leq 10 \\
x_{1}, x_{2} \geq 0 \text { and integers. }
\end{gathered}
$$

By using ranking function of $\left(y_{01}\right), \mathfrak{R}\left(y_{01}\right)=\frac{1}{2}\left(a^{l}+a^{u}+\frac{1}{2}(\beta-\alpha)\right), \mathfrak{R}\left(y_{01}\right)=-7.25, \mathfrak{R}\left(y_{02}\right)=-9$. Since the problem

\begin{tabular}{|c|c|c|c|}
\hline & $\tilde{t_{1}}$ & $\tilde{t_{2}}$ & R.H.S \\
\hline $\mathrm{Z}$ & $(-8,-5,5,2)$ & $(-10,-6,6,2)$ & $\tilde{0}$ \\
\hline$x_{1}$ & -1 & 0 & 0 \\
\hline$x_{2}$ & 0 & -1 & 0 \\
\hline$S_{1}$ & 2 & $(3)$ & 6 \\
\hline$S_{2}$ & 5 & 4 & 10 \\
\hline$S_{c 1}$ & 0 & $((1))$ & 2 \\
\hline
\end{tabular}

is maximum, and by using simplex algorithm the interring variable is $\tilde{t}_{2}$ and leaving variable is $\left(S_{1}\right)$. To find the new table first we find the elements of the $\operatorname{row}\left(S_{c 1}\right)$ as follows

$$
S_{c_{1}}=\left(\left[\frac{2}{3}\right],\left[\frac{3}{3}\right],\left[\frac{6}{3}\right]\right)=(0,1,2)
$$

Second, the pivot element is ((1)),we apply simplex algorithm to find the new table as follows:

\begin{tabular}{|c|c|c|c|}
\hline & $\tilde{t_{1}}$ & $\tilde{t}_{2}$ & R.H.S \\
\hline $\mathrm{Z}$ & $(-8,-5,5,2)$ & $(6,10,2,6)$ & $(12,20,4,12)$ \\
\hline$x_{1}$ & -1 & 0 & 0 \\
\hline$x_{2}$ & 0 & 1 & 2 \\
\hline$S_{1}$ & 2 & -3 & 0 \\
\hline$S_{2}$ & $(5)$ & -4 & 2 \\
\hline$S_{c 1}$ & $((1))$ & -1 & 0 \\
\hline
\end{tabular}

$$
S_{c_{2}}=\left(\left[\frac{5}{5}\right],\left[\frac{-4}{5}\right],\left[\frac{2}{5}\right]\right)=(1,-1,0)
$$

The pivot element is $((1))$, we apply simplex algorithm to find the table (3), since

$$
\Re\left(y_{01}\right)=\frac{1}{2}\left(13+\frac{1}{2}(5-2)\right)=\frac{1}{2}\left(13+\frac{3}{2}\right) \geq 0
$$

and

$$
\Re\left(y_{02}\right)=\frac{1}{2}\left(5-2+\frac{1}{2}(8-7)\right)=\frac{1}{2}\left(3+\frac{3}{2}\right) \geq 0
$$

Therefore the optimal fuzzy integer solution is $\tilde{z}(12,20,4,12), x_{1}=0 x_{2}=2$. Note: the above problem is solved in paper [2] and found the optimal non integer solution is $\tilde{Z}\left(\frac{90}{7}, \frac{148}{7}, \frac{32}{7}, \frac{90}{7}\right), X_{1}=\frac{10}{7}, X_{2}=\frac{6}{7}$. 


\section{Example}

In this example we treat with (CFLIPP).

$$
\min \tilde{z}=(-3,-1,2,2) \tilde{x_{1}}+(-6,1,-1,-1) \tilde{x_{2}} .
$$

Subject to

$$
\begin{aligned}
& (0,1,2,2) \tilde{x_{1}}+(1,2,3,3) \tilde{x_{2}} \leq(4,6,3,3) . \\
& (1,2,3,3) \tilde{x_{1}}+(0,1,2,2) \tilde{x_{2}} \leq(1,3,2,2) .
\end{aligned}
$$

$\tilde{x_{1}}, \tilde{x_{2}} \geq 0$ and integers. Here we solve this problem directly by using linearity property of ranking function transformation all the fuzzy constraint and restriction into the crisp constraints and all (LPP) can be written as

$$
\max \mathfrak{R}(\tilde{z})=(\mathfrak{R}(1,2,3,2)) \bigotimes \mathfrak{R}\left(\tilde{x_{1}}\right)+(\mathfrak{R}(2,3,1,1)) \bigotimes \mathfrak{R}\left(\tilde{x_{2}}\right) .
$$

Subject to

$$
\begin{aligned}
& (\Re(0,1,2,2)) \bigotimes \Re\left(\tilde{x_{1}}\right)+(\Re(1,2,3,2)) \bigotimes \Re\left(\tilde{x_{2}}\right) \leq(\Re(1,5,4,2)), \\
& (\Re(1,2,3,2)) \bigotimes \Re\left(\tilde{x_{1}}\right)+(\Re(0,1,2,2)) \bigotimes \Re\left(\tilde{x_{2}}\right) \leq(\Re(2,6,1,2)),
\end{aligned}
$$

$\mathfrak{R}\left(\tilde{x_{1}}\right), \mathfrak{R}\left(\tilde{x_{2}}\right) S \geq 0$, since if $\tilde{a}=\left(a^{l}, a^{u}, \alpha, \beta\right)$ then

$$
\Re(\tilde{a})=\frac{1}{2}\left(a^{L}+a^{u}+\frac{1}{2}(\beta-\alpha)\right) .
$$

Therefore the new problem is as below:

$$
\max \Re(\tilde{Z})=\frac{5}{4} x_{1}+\frac{5}{2} x_{2}
$$

Subject to

$$
\begin{aligned}
& \frac{1}{2} x_{1}+\frac{5}{4} x_{2} \leq \frac{5}{2} \\
& \frac{5}{4} x_{1}+\frac{1}{2} x_{2} \leq \frac{17}{4}
\end{aligned}
$$

$$
x_{1}, x_{2} \geq 0
$$

and integers. Now by using development integer simplex table we find the optimal as follows,

$$
\left(\begin{array}{cccc} 
& \tilde{t_{1}} & \tilde{t_{2}} & R . H . S \\
Z & \frac{5}{4} & 0 & 5 \\
x_{1} & 1 & -2 & 0 \\
x_{2} & 0 & 1 & 2 \\
S_{1} & -\frac{1}{2} & -\frac{1}{4} & 0 \\
S_{2} & -\frac{5}{4} & 2 & \frac{13}{4}
\end{array}\right) .
$$

The optimal integer solution is $Z=5, x_{1}=0, x_{2}=2$. 


\section{Inference}

This technique is considered one of the new and best ways to find the solution of optimal (FILPP) and completely fuzzy Integer Linear Programming Problems (CFILPP) by using a linear ranking function, since the ease of calculation and speed in the solution and the lack of number of tables of solution compared to other methods.

\section{Competing interests}

The authors declare that they have no competing interests.

\section{Authors' contributions}

All authors have contributed to all parts of the article. All authors read and approved the final manuscript.

\section{References}

[1] P.Pandian , M.Jayalakshmi , A new method for solving integer linear programming problems with fuzzy variables , Applied Mathematical sciences, vol .(4) , no (20), pp. 997- 1004 , 2010.

[2] N. M.Amiri , S.H.Nasseri, A. Yazdani , Fuzzy primal simplex Algorithms for solving fuzzy linear programming problems , Iranian Journal of operation research, vol.(1), no.(2), pp. 68-84, 2009.

[3] S.H.Nasseri, E.Ardil , simplex method for fuzzy variable linear programming problems , International Journal of mathematical , computational, physical and Quantum Engineering vol.(3), no(10), 2009.

[4] I.H.Alkanani , F.A.Adnan, Ranking Function Method for Solving Fuzzy linear programming problems , Mathematical theory and modding, vol.(4), no.(4), 2014.

[5] M.Jayalakshmi , P.Pandian , A New method for finding an optimal fuzzy solution for fully fuzzy linear programming problems , International Journal of Engineering Research and Applications , vol.(2), Issue(4), pp.247-254 , 2012.

[6] S.S.Roseline, E.C.Henry Amirthajaj, Different strategies to solve fuzzy linear programming problems, science and technology , 4(5), 2012.

[7] A.Kumar , P.Singh , J.Kaur, Generalized Simplex algorithm to solve fuzzy linear programming problems with Ranking of Generalized fuzzy Numbers, Turkish Journal of fuzzy systems vol.(1) , no.(2) , pp.80-103 , 2010.

[8] Someshwar Siddi, Dr. Y. Raghunatha Reddy, Solution of integer linear programming problems with triangular fuzzy numbers, 6(3), March, 2017, International journal of engineering sciences \& research technology.

[9] D. Stephen Dinagar and M Mohamed Jeyavuthin, Fully Fuzzy Integer Linear Programming Problems Under Robust Ranking Techniques , 6(3), 2018 , pp.19-25 , International journal of mathematical and its applications . P.Rajaraes , 